\title{
La aplicación de la formación para el análisis sobre las salas VIP del
}

\section{Aeropuerto Ecológicos}

The application of the training for the analysis on the VIP lounges of the Ecological Airport

A aplicação do treinamento para análise nas salas VIP do Aeroporto Ecológico

\section{Lolay Burbano Mosquera}

Magister. Docente del Instituto Tecnológico Superior Vicente Rocafuerte.

Lolitatburbano@hotmail.com, Guayaquil, Ecuador, https://orcid.org/0000-0002-3604-1651

\section{Luis Triguero Litardo}

Estudiantes de Hotelería y Turismo. Docente del Instituto Tecnológico Superior Vicente Rocafuerte, Juan_triguero14@hotmail.es Guayaquil, Ecuador, https://orcid.org/0000-00030725-2625

\section{María Valeria Costa Sosa}

Licenciada. Docente del Instituto Tecnológico Superior Vicente Rocafuerte. mcosta@itsvr.edu.ec, Guayaquil, Ecuador, https://orcid.org/0000-0001-5785-1680

Recibido 23 enero 2017 - Aceptado 4 marzo 2017

Formación docente - revista iberoamericana de educación http://www.revista-iberoamericana.org/index.php/es/index

https://creativecommons.org/licenses/by/4.0/deed.es e-ISSN: $2737-632 X$

Vol - 1 No. 1, enero - diciembre 2018

Pags 1-18

Resumen La odisea de construir un aeropuerto ecológico se hace posible al leer acerca de esta magna construcción que es la primera terminal aérea en el mundo en ser autosustentable y autosostenible, por todos sus materiales utilizados para su reconstrucción, esta por demás enunciar de que los pasos a seguir para la remodelación de sus Salas VIP de Arribos y Partidas del Aeropuerto Seymour de Baltra son similares a los de su construcción en general. Tomando en cuenta un análisis de todos sus materiales, el mantenimiento de todo el mobiliario y demás instalaciones, las mismas que demuestran la preocupación por la preservación de las Islas Galápagos de 
parte de la empresa ECOGAL S.A. como Patrimonio Cultural de la Humanidad, a quien le fue concedida la administración de este interesante lugar. Incluimos también los resultados, gráficos, comparaciones y conclusiones, para que cuando el lector se inmersa en este desafío de leer acerca de este proceso pueda ampliar más sus conocimientos acerca de la remodelación de las Salas VIP de este importante aeropuerto. Toda la información adquirida ha sido recopilada de fuentes seguras que dan certeza de lo que se expone en este artículo, las mismas que pueden ser comprobables a través de su bibliografía, citas y demás vínculos expuestos.

Palabras clave: Aeropuerto ecológico, Sala VIP, Sostenibilidad.

Abstract The odyssey of building an ecological airport is made possible by reading about this great construction that is the first air terminal in the world to be self-sustaining and self-sustaining, due to all the materials used for its reconstruction, it is worth mentioning that the steps to continue to remodel their Arrivals and Departures VIP Lounges at Baltra Seymour Airport are similar to their general construction. Taking into account an analysis of all its materials, the maintenance of all the furniture and other facilities, which demonstrate the concern for the preservation of the Galapagos Islands by the company ECOGAL S.A. as a Cultural Heritage of Humanity, to whom the administration of this interesting place was granted. We also include the results, graphs, comparisons and conclusions, so that when the reader is immersed in this challenge of reading about this process, they can further expand their knowledge about the remodeling of the VIP Lounges of this important airport. All the information acquired has been compiled from secure sources that give certainty of what is exposed in this article, the same that can be verified through its bibliography, citations and other exposed links. 
Keywords: ecological airport, VIP Lounge, Sustainability.

Resumo. A odisséia de construir um aeroporto ecológico é possível graças à leitura desta grande construção, que é o primeiro terminal aéreo do mundo a ser auto-sustentável e auto-sustentável, devido a todos os materiais utilizados na sua reconstrução, vale ressaltar que os passos para continuam a reformar suas salas VIP de chegadas e partidas no aeroporto de Baltra Seymour são semelhantes à sua construção geral. Levando em consideração uma análise de todos os seus materiais, a manutenção de todos os móveis e outras instalações, que demonstram a preocupação com a preservação das Ilhas Galápagos pela empresa ECOGAL S.A. como Patrimônio Cultural da Humanidade, a quem foi concedida a administração deste lugar interessante. Também incluímos os resultados, gráficos, comparações e conclusões, para que, quando o leitor estiver imerso nesse desafio de ler sobre esse processo, ele possa expandir ainda mais seus conhecimentos sobre a reforma das salas VIP deste importante aeroporto. Todas as informações adquiridas foram compiladas de fontes seguras que garantem o que é exposto neste artigo, o mesmo que pode ser verificado através de sua bibliografia, citações e outros links expostos.

Palavras-chave: Aeroporto ecológico, Lounge VIP, Sustentabilidade.

\section{INTRODUCCIÓN}

Las Islas Galápagos declaradas en 1979 por la Unesco como Patrimonio de la Humanidad (Macias, 2012), posee una de las mayores riquezas en flora y fauna debido a su gran Reserva de la Biosfera, por lo cual sus habitantes en conjunto con el gobierno se han esmerando en el cuidado de estas islas al proveer un ambiente totalmente saludable para preservación de ellas, en tan preciado lugar se ubica el Aeropuerto Seymour de Baltra, considerado como el primer Aeropuerto Ecológico del mundo. Tuvo sus inicios como base militar estadounidense, la cual al finalizar la II Guerra Mundial el Presidente 
José María Velazco Ibarra ordenó tajantemente el desalojo de los soldados norteamericanos, quienes en represalias a aquel mandato destruyeron y arrojaron al mar gran parte de las instalaciones de la base que posteriormente fue remodelada y retomada por el gobierno ecuatoriano; luego entregada a las fuerzas aéreas y la marina.

Esta gran obra implementada en la región insular ha ganado muchos premios internacionales que la han acreditado a ser uno de los mejores en el mundo. Uno de las condiciones para acceder al premio de Certificación LEDD GOLD por su diseño y construcción en las que destacan la reducción del consumo energético y la producción de energía limpia (solar y eólica) en un 100\%, la recuperación de áreas afectadas y la reforestación de flora endémica, la iluminación y ventilación natural adecuada en todos los espacios, la reducción del consumo de agua, tratamiento y reutilización de aguas además de reutilizar en un $80 \%$ de material del antiguo aeropuerto. Además de esto obtuvo una Certificación Nivel 4 AIRPORT CARBON ACCREDITED NEUTRALITY que se inició en el 2014 y desde entonces hasta la fecha se ha logrado reducir las emisiones de gases de efecto invernadero.

El sector aeronáutico es uno de los responsables de la contaminación ambiental (MAE, 2015). Sin embargo, en la actualidad dentro de una sociedad cada día más globalizada este elemento de conectividad es indispensable. Las operaciones básicas que realiza un avión para volar tienen un gran impacto ambiental. Pero, se han venido corrigiendo procedimientos para reducir la contaminación ambiental que produce el sector aeronáutico. Es por ello que durante la última década, el sector ha realizado ciertos avances en mejorar su comportamiento medio ambiental. Por ejemplo se han aplicado medidas para una reducción en sus costes económicos energéticos. Por lo anterior, unido a la gran cantidad de emisiones GEI (Gases de Efecto Invernadero), se está impulsando grandes cambios en el sector aeronáutico para convertirlo en 
sector más sostenible (Plataforma de Conocimiento sobre Construcción Ambientalmente Sostenible de Infraestructura en América Latina y el Caribe).

Este aeropuerto es de vital importancia no solo por lo antes mencionado sino también porque todas sus instalaciones están hechas con la finalidad de respetar el medio ambiente y causar el menor impacto posible a la naturaleza que se encuentra en sus alrededores además de ser un medio autosustentable y auto sostenible, (ECOGAL S.A, 2017).

La Sociedad concesionaria que es la responsable de administrar y operar el Aeropuerto Seymour de Baltra, es una empresa que pertenece a Corporación América llamada ECOGAL S. A. y a la que el Gobierno Ecuatoriano le asignó la concesión de este, durante 15 años.

La estructura exterior utiliza un espacio de $6.000 \mathrm{~m} 2$, su techo en forma de sombrilla llega hasta los $10.000 \mathrm{~m} 2$. Su estructura modernizada se sostiene por tuberías que fueron recuperadas de algunos campos de extracción petrolera en la Amazonía del Ecuador. Cuenta con paredes de color claro y cuenta con una superficie tipo ondas, que deja ingresar la luz natural, aunque de manera indirecta. Esta terminal se encuentra ubicada en un ángulo de cuarenta y cinco grados, a una distancia muy confiable de la pista, que es lo estipulado en los requisitos internacionales que tratan estos temas de seguridad, haciendo que las emanaciones de gases de los aviones no ingresen al edificio y al mismo instante reduciendo niveles de ruido dentro del espacio del aeropuerto.

De la iluminación y la ventilación se encargan el Parque Eólico junto con los paneles fotovoltaicos propios del aeropuerto, situados en las camineras, brindan el $100 \%$ de la energía renovable que utiliza dicha instalación. Los ventanales grandes apoyados en dos sistemas de claraboyas ubicadas a la altura de la sala de chequeo y en la parte central del edificio; funcionan como 
una entrada de circulación de aire y también de luz. El aire se va renovando y extrayendo constantemente a través de unas lucarnas que están en el techo. Conforme el aire comienza a subir su temperatura los louvers mecánicos automáticamente la nivelan mediante sensores.

La planta de agua desalinizadora con la que cuenta esta terminal, recoge el agua del mar para tratarla y luego llevarla a los tanques de almacenamiento ubicados junto al edificio principal. Una vez que esta agua es desalinizada, ingresa a la terminal por tuberías y una vez que es usada, se vuelve a recolectar, se trata y se recicla, evitando de esta manera la generación de agua residual y ayudando para el ahorro de agua. El agua de los inodoros y de los lavamanos se recicla y se reutiliza, para usarla en los sanitarios. El color (turbio) de esta agua es resultado del proceso de tratamiento de reutilización sin tener ninguna relación a suciedad, deterioro o contaminación. No generan ninguna contaminación también, los urinarios; ya que son secos, no utilizan agua, ni agua de deshecho.

Se utilizaron materiales reutilizados de antiguas canteras, como los agregados pétreos. Como revestimiento de los muros externos de la terminal, se usó piedra volcánica, la misma que la acomodó con el entorno, logrando así un menor impacto al medio ambiente. Todo lo que tiene que ver con la madera y las estructuras metálicas fueron reutilizadas de la antigua terminal. Las caminerias y los accesos vehiculares, están hechas de puro hormigón por ser un material más amigable con el ambiente, evitando de esta forma el excesivo calentamiento y el efecto de isla de color. La señalética del aeropuerto es de acero inoxidable, totalmente ecológico y en su impresión se usaron tintas de vinil eco-solventes.

Por esto y más; este Aeropuerto Ecológico de Galápagos, ha sido acreditado como el mejor del mundo. 
Una vez que Aerolíneas Avianca, entró con sus cinco vuelos semanales a este aeropuerto, a finales del año 2003; hubo un aumento de pasajeros tanto de arribos como de partidas, lo que empezó a ocasionar una saturación en sus salas VIP, las mismas que se vieron afectadas por falta de espacio. Ante esta limitación Ecogal. S. A. Tomó la decisión de aumentar más espacio en dichas Salas; comenzando así, un proyecto de expansión tanto en la de Arribos como en la de Partidas.

En el transcurso del estudio arquitectónico y de diseño, se fue encontrando algunos limitantes, entre ellos una mampara ubicada en el área donde se quería hacer tal remodelación, ante lo cual, se dispuso que en vez de hacerse dos salas grandes, se hicieran 18 salas pequeñas, tipo sala de estar de una casa acogedora, bajo el slogan: "siéntete como en casa". Estas salidas debían estar tan confortablemente posibles para que el turista pueda sentirse como dice el slogan; como en casa.

Ahora bien, en artículos anteriores a este, hemos visto algunos análisis y estudios del aeropuerto en sí, más no de sus Salas VIP (arribos y partidas).

Por lo que en este artículo vamos a analizar los recursos que han sido utilizados en esta remodelación, dar a conocer como fue este proceso y por último informar acerca de su mantenimiento.

Esta investigación se efectuó en la ciudad de Guayaquil, y el tiempo que se empleo fue un mes, la razón por la cual es que la persona que nos ayudó o proveyó de la información reside en el Cantón Guayas, Provincia de Guayaquil.

\section{MATERIALES Y MÉTODOS}

Este análisis se basará en la renovación de las salas de preembarque y arribos del Aeropuerto Seymour de Baltra (Galápagos), donde se contó con la colaboración de la arquitecta encargada de este proyecto, por medio de una 
entrevista personal, en la cual detalla paso a paso el proceso de dicha renovación y proveyendo planos, fotografías y más materiales para esta acción.

Planta arquitectónica de salas VIP arribos:

Para esta acción se contó con algunos materiales existentes y otros que había que comprar, de los cuales nombramos a continuación: sofá 4 puestos, sofá 3 puestos, bancos de 1 puesto, sillas de bar, mesas de centro, mesas de bufet y exhibidos de bebidas.

Proceso de remodelación de las salas a-a; b-b; c-c y d-d salón VIP arribos.

Uno de los materiales usados en esta remodelación es la piedra volcánica, originaria de la isla que será usado en las divisiones de paletas de pino laqueado, con bandeja de latón. Divisiones de cruz, realizadas con pallets de pino laqueado. El bar fue fabricado con pared de pallets de pino laqueado también; se protegió la encimera con una placa de vidrio en el mesón y se colocaron lámparas colgantes de colores fuertes para que combine con los colores de los cojines. El cielo falso o tumbado fue fabricado con pallets dispuesto como tablero de ajedrez.

Las mesas de centro y auxiliares fueron fabricados con pallets de pino, laqueado con laca transparente mate más oscura (técnica de envejecido) y las lámparas colocadas en las mesas auxiliares fueron de un armazón metálico ligero con pantalla de tela y yute. Mampara de aluminio y vidrio con lámina decorativa reutilizado. Muebles de ratán reutilizados de las antiguas salas VIP los ya existentes; y los que se mandó a fabricar fueron de pallets con acabado laqueado transparente oscuro y colchonetas de cuero blanco y para armonizar se colocaron almohadones de colores vivos. Se utilizó caña guadua existente la cual se colocó como elemento colgante con cables de acero; a los cuales se les quemó los nudos y se laqueó. 
La pared fue revestida de piedra volcánica de diferentes tamaños, y los muebles fabricados con pallets. En cada una de las salas se diseñó la misma estructura. (Ver anexo imagen $N^{\circ} 4$ )

Proceso de remodelación de las salas e-e, f-f, g-g, h-h salón VIP partidas. El proceso que fue utilizado en las salas VIP de arribos, se utilizó en las salas de partidas y su diseño igualmente estructurado, las divisiones entre las salas se mantuvieron de la misma forma en la que estaban antes, siendo reutilizadas en cierta forma las cañas guaduas. Cabe destacar ciertos detalles, como la reutilización de los muebles de las antiguas salas VIP y los que faltaron fueron hechos del mismo diseño y con la misma clase de material de los otros existentes.

Se elevó un tumbado descolgado de gypsum en la sala VIP de partidas. Como decoración central, reutilizando las tiras de madera de las pérgolas del antiguo salón de partidas; el mismo que fue sujeto a la estructura de cubierta mediante cables de acero. En la estructura metálica que fue revestida con placas de gypsum, la misma que contiene del cielo falso, se colocó un televisor.

Mantenimiento del mobiliario de salas VIP de partidas y arribos del aeropuerto Seymour de Baltra. Barros-Bastidas, C., \& Turpo, O. (2020).

Este artículo tiene como finalidad, recomendar las acciones a tomar para darle mantenimiento de las salas VIP del aeropuerto, y puedan mantenerse en buen estado a lo largo del tiempo. Aunque como dificultad se presenta la condición climática de la isla y el uso de los muebles por parte de los empleados de las salas y pasajeros, por lo tanto es necesario tomar algunas precauciones para que los mantenimientos necesarios sean cada vez menos frecuentes en la etapa lluviosa, que es abundante pudimos constatar que la lluvia ingresa por los ventanales de las salas, mojando los muebles y en algunos casos (según comentarios del personal que atiende las salas VIP) se inundan las salas. Por lo que el inmobiliario estaría expuesto a un grave daño; ya que este, no está 
diseñado para soportar el tipo de humedad provocado por la lluvia. Para aminorar esta situación (de darse se recomendó implementar un sistema de cortinas enrollables de lona pesada, las que se desplegarían cuando lloviera torrencialmente, o en su defecto se procedería a retirar los cojines y colchonetas a un sitio seco y cubrir los muebles con una manta con un plástico. Que se coloque cartón, playwood etc., a la superficie de pallet que tiene contacto con las colchonetas de cuerina blanca, así se evita que la humedad se concentre y que haga presencia de Moo. Aunque si el moho aparece la limpieza debe ser diaria, seguir colocando el cartón y orear cuando estén en desuso cae recalcar, que el polvo rojo que emite la isla, debe retirarse de los muebles o de las instalaciones por lo menos una vez por semana de cada elemento que forma parte de la decoración de las salas VIP.

Barros Bastidas, C., \& Turpo Gebera, O. (2018). En vista de que la acumulación de este polvo, a pesar de no dañar el mueble confinado con la humedad y grasa, formaría una película difícil de retirar dándole más aspecto al mueble.

En cuanto al uso de los muebles constatamos que los pasajeros a menudo colocan los pies sobre las mesas de centro por lo que las mismas, estarían expuestas consta mente a los rayones de los zapatos. En tal caso y como se sugirió para proteger los encimemos d los bares; también recomendamos que se coloque sobre las mesas de pallet vidrios de mínimo de $6 \mathrm{~mm}$ con ventosas para evitar que resbale el vidrio, dando como mensaje subliminar, de no subir los pies y así proteger la superficie de los pallet tanto de los rayones de los zapatos, arrastre de objetos así como los derramamiento de bebidas.

Aunque los muebles de bebida, tiene todos los tratamientos propios de un mueble, se les debe cuidado con los derrames o goteos de líquidos como se lo hace a diario. La máquina de café tiene un constante goteo, por lo cual se extendieron los encimemos para poder colocar un plato que pueda recoger 
este goteo. Para la máquina de jugos (aunque esta provista de una bandeja anti derrame) es necesario limpiarla a diario; puesto que las salpicaduras de jugos pueden manchar permanentemente la madera.

Recordando que el concepto de diseño de las salas VIP es la sustentabilidad; razón por la cual se usaron placer para elaborar o fabricar la mayoría de los muebles y darles la impresión de que el pallet es viejo y que se reutilizo. Sin embargo la madera de los pallet de exportación es pino, por lo que se considera un material grasoso, solo podrá mantenerse en el tiempo cumpliendo con las indicaciones que se describen en este artículo.

Rodríguez Morales, A., Barros Bastida, C., \& Milanés Gómez, R. (2019).

Mencionaremos algunas sugerencias para tratar todo el mobiliario de materiales distintos al pallet, los cuales; detallamos a continuación: los muebles fabricados con pallet de madera pino tratado, únicamente consiste en retirar el polvo con un paño humedecido y bien exprimido (no empapado de agua y sin ninguna clase de químico alguno). Estos elementos no requerirán de uso de aceite para dar brillo, ya que la laca catalizada final; con que se laquearon; les da cierto brillo semimate. Si se llegan a usar aceites para dar brillo, estos pueden generar manchas o huellas por el secado o aplicación irregular. Ninguno de estos elementos debe estar a la intemperie solar ni de lluviosa.

El mantener estos muebles bien cuidados depende de la limpieza de por lo menos, una vez a la semana y consiste en lo siguiente: después del primer año de instalación y por causa de la humedad a la que están expuestos durante el traslado de los mismos a la isla e baltra, se procede; al lijado y la aplicación de una mano de laca nitro celulosa semibrillante. Este mantenimiento con su tratamiento se recomienda una vez cada dos años.

Mantenimiento de elementos fabricados en pallets y playwoods, ubicado en los exteriores (muebles del bar, sillas altas, mesas auxiliares y de centro y división 
de bandejas de piedra de salas VIP partidas). Tapia-León, M., Rivera Villalta, M. D. C., Luján-Mora, S., \& Barros Bastidas, C. I. (2017).

A los que se les aplicó poliéster antes de la mano final de acabado (bar de exteriores, encimemos de todos los bares, silla altas y mesas auxiliares y de centro), deberán limpiarse de igual manera con paños humedecidos y sin ningún químico de limpieza o aceites para abrillantar, su mantenimiento consiste en una mano de poliéster, más la mano de acabado semi mate, una vez cada dos años, ya que este mobiliario se encuentra a la intérprete.

En el caso de los pallets y encimemos de todos los bares; así como hemos constatado su uso (arrastre de objetos, zapatos sobre mesas, posibilidad de derrames de líquidos) se sugirió que se le coloque un vidrio de mínimo 6 mm con ventosas, y de ser así el mantenimiento de estos elementos podría extenderse a una vez cada tres años.

Para lo que es la división de bandera de piedra volcánica, la misma que se encuentra fuera de la sala se deberá aplicar una mano de laca catalizada una vez al año.

Elementos fabricados en cartón trochado:

Esta clase de muebles también debe limpiarse con un paño humedecido sin el uso necesario de aceites y demás químicos. Bajo ningún concepto no deben colocarse exteriores ya que no podrán soportar la exposición directa del sol y la lluvia. El retiro del polvo de la isla debe hacerse una vez a la semana.

En época lluviosa se recomienda que los muebles de mimbre y cartón trocado ubicados en el exterior deben ser ingresados a las salas de partidas, ya que a pesar de estar protegidos bajo cubierta, podrían mojarse por la lluvia que en muchas veces es multidireccional. Como posible alternativa esta podría ser cubrir los muebles con un manta de plástico cuando este inhabilitado. 
Si ocurre decoloración de esto elementos, sugerimos que se realice cada dos años un laqueado de nitrocelulosa con color para que su durabilidad sea de más de quince años o más.

Elementos fabricados en madera de roble (mesas auxiliares, tableros de mesa de bufet y marco de cuadro de ambas salas).

A todos estos muebles fabricados en madera de roble se les debe limpiar el polvo de la isla al menos una vez a la semana. A las superficies horizontales; como las mesas (de bufet y auxiliares), que están expuestas a rayones debido a la rastra de objetos sugerimos que se ligue y coloque una mano de laqua catalizada cada dos años en el caso de las mesas auxiliares y una vez al año en el ano de las mesas de bufet (si es que ocurren los rayones).

En el cuadro mural que está en la sala de arribos pudimos constatar rastros de humedad por lo que si llegara a mojarse con la lluvia de marco de madera de roble; como mantenimiento se le debe aplicar una laca catalizada cada año Colchonetas y cojines:

Con respecto al material de acabado de las colchonetas (cuerina expandible), deben limpiarse con paño humedecido para retirar el polvo y podría aplicarse algún tipo de químico para aplicar manchas (SIF Crema) que no deteriore o descascare la cuerina obviamente se retira el químico con un paño humedecido al final.

Los cojines (confeccionados en telas vistosas de micro fibra) pueden lavarse con agua y jabón de uso doméstico, si llegara a usarse.

Los cojines a rayas deben lavarse únicamente en seco (como lo indica el fabricante en la etiqueta). Como importante recomendación preferiblemente deberían enviarse a Guayaquil-ecuador continental por vía aérea para su lavado; dividiéndolo en dos partes para que las salas no se queden sin cojines. Marco teórico 
La idea es de Corporación América, un "Holding" argentino que en su portafolio muestra la intervención de más de 50 aeropuertos y cuenta con alta experiencia en la edificación de estas estructuras (MUNDO CONSTRUCTOR, 2016). La organización junto a su equipo de arquitectos de nacionalidad inglesa, e hicieron un diseño sustentable que siguió, desde los inicios, los parámetros exigidos por US GREEN BUILLDING COUNCIL, con el objetivo de obtener la certificación Leed ORO.

Según el grupo de arquitectos, se basó e inspiró en los recursos naturales de la isla. La ubicación de la terminal aérea responde a criterios como la posición del sol y los vientos de la isla. También, se consideró la conservación de plantas endémicas, no podían ser movidas y se las adaptó como plantas movidas.

\section{RESULTADOS}

En el análisis propuesto se ha podido evidenciar que los materiales usados en la remodelación de las salas VIP del Aeropuerto Seymour de Baltra (Galápagos), el 50\% ha sido exportado desde la ciudad de Guayaquil (Ecuador Continental) pallets de pino laqueado y el 30\% es de material endémico de la isla y un $20 \%$ ha sido reutilizado de ciertas dependencias del aeropuerto en la isla. Todo este material es netamente ecológico lo cual no afecta de ninguna manera la estabilidad de la isla y principal mete su medio ambiente, garantizando así; su sostenibilidad y sustentabilidad de la que se sirve este aeropuerto.

Tabla $N^{\circ} 1$ Estadísticas de visitantes

\begin{tabular}{llll} 
AÑO & EXTRANJEROS & NACIONALES & TOTALES \\
\hline $\mathbf{2 0 1 2}$ & 125.059 & 55.772 & 180.831 \\
$\mathbf{2 0 1 3}$ & 132.119 & 72.276 & 204.395
\end{tabular}




$\begin{array}{llll}\mathbf{2 0 1 4} & 149.997 & 65.694 & 215.691 \\ \mathbf{2 0 1 5} & 154.304 & 70.451 & 224.755 \\ \mathbf{2 0 1 6} & 149.766 & 68.599 & 218.365 \\ \mathbf{2 0 1 7} & 84.103 & 35.754 & 119.857\end{array}$

Autoría propia: Luis Triguero Litardo

Lista de materiales que se utilizaron en la remodelación del Aeropuerto

Seymour de Baltra:

Tabla $N^{\circ} 2$ Lista de materiales para la remodelación Aeropuerto Seymour de Baltra

\begin{tabular}{|c|c|c|c|}
\hline $\begin{array}{l}\text { Materiales } \\
\text { endémicos }\end{array}$ & Porcentaje & $\begin{array}{l}\text { Materiales traídos } \\
\text { del ecuador } \\
\text { continental }\end{array}$ & Porcentaje \\
\hline Piedra volcánica & $25 \%$ & Pallets de pino & $4 \%$ \\
\hline $\begin{array}{l}\text { Estructura } \\
\text { metálica }\end{array}$ & $20 \%$ & Vidrio & $4 \%$ \\
\hline $\begin{array}{l}\text { Mampara de } \\
\text { aluminio }\end{array}$ & $9 \%$ & Muebles de mimbre & $9 \%$ \\
\hline \multirow[t]{6}{*}{ Muebles de ratán } & $10 \%$ & Caña guadua & $9 \%$ \\
\hline & & Pérgolas & $2 \%$ \\
\hline & & Gypsum & $3 \%$ \\
\hline & & Madera de cedrela & $2 \%$ \\
\hline & & Bandejas de latón & $2 \%$ \\
\hline & & Cable de acero & $1 \%$ \\
\hline
\end{tabular}

El presente grafico ilustra el porcentaje de materiales que fueron reutilizados para el proceso de remodelación de las Salas VIP del Aeropuerto Seymour de Baltra. 


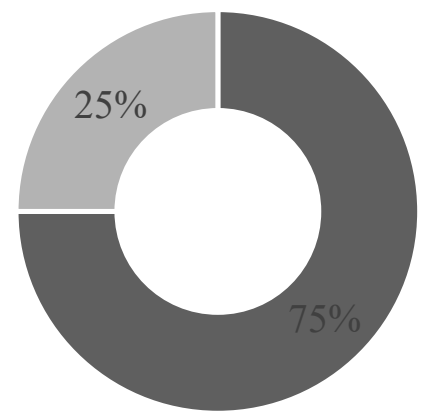

- MATERIALES

DE LA

ANTIGUA

TERMINAL

- MATERIALES

TRAIDOS DEL

ECUADOR

CONTINENTAL

Gráfico 1: Porcentaje de visitantes al aeropuerto de baltra Autoría propia: Luis Triguero Litardo

\section{CONCLUSIONES}

La preservación del medioambiente, es tarea de todos, y en este tiempo es cuando más se está trabajando en ello, y; prueba de esto es el Aeropuerto Seymour de Baltra y todas sus instalaciones, incluso en la remodelación de sus salas VIP, todos los materiales que se han usado en este proyecto son reutilizados en su mayoría, y los que no; son netamente ecológicos, evidenciando así; que la empresa ECOGAL S. A. encargada de la administración de esta terminal, se encuentra totalmente comprometida con la preservación de la biodiversidad de las Islas Galápagos. La mayoría de las páginas web y los artículos leídos refieren este importante tema resaltando el alcance a nivel mundial que tiene la construcción de este aeropuerto y todas sus instalaciones.

\section{REFERENCIAS}

Ambiente, M. d. (2015). Medio Ambiente. Quito, Pichincha, Ecuador. Obtenido de http://www.ambiente.gob.ec/wpcontent/uploads/downloads/2012/09/LEY-DE-PREVENCION-YCONTROL-DE-LA-CONTAMINACION-AMBIENTAL.pdf 
Arq. Vanessa Alvarez Del Pozo de Haro. (2012). Descripcion de la rediseñacion de las salas VIP del Aeropuerto Seymour de Galápagos. Baltra, Islas Galápagos, Ecuador Continental . Recuperado el 17 de Enero de 2017

Barros-Bastidas, C., \& Turpo, O. (2020). La formación en investigación y su incidencia en la producción científica del profesorado de educa- ción de una universidad pública de Ecuador. Publicaciones, 50(2), 167-185. doi:10.30827/publicaciones.v50i2.13952

Barros Bastidas, C., \& Turpo Gebera, O. (2018). Factors influencing the scientific production of university professors: a systematic review .Pensamiento Americano, 11(22). https://doi.org/10.21803/pensam.v11i21-1.276

Certificaciones. (2012). premios obtenidos del primer aeropuerto ecologico del mundo. Baltra, Galápagos, Ecuador. Obtenido de http://www.ecogal.aero/certificaciones-y-premios

ECOGAL S.A. (7 de Junio de 2017). Aeropuerto Ecologico del Mundo. (L. Vélez, Ed.) AeroMundo Magazine, 3. Recuperado el 21 de Marzo de 2018, de https:/www.aeromundomagazine.com/aeropuertoecologico-de-galapagos-el-nuevo-encanto-de-las-islas/

Macias, C. (2012). Primer Aeropuerto Ecológico del Mundo. Guayaquil, guayas, Ecuador. Obtenido de https://es.linkedin.com/pulse/elprimer-aeropuerto-ecologico-del-mundo-se-encuentra-carla-macias

MUNDO CONSTRUCTOR. (06 de Septiembre de 2016). Baltra: un aeropuerto responsable con el archipiélago. Obtenido de http://www.mundoconstructor.com.ec/index.php/construccion/comer 
cial/689-baltra-un-aeropuerto-responsable-con-el-

archipi\%C3\%A9lago

Rodríguez Morales, A., Barros Bastida, C., \& Milanés Gómez, R. (2019).

Profesionalización docente y formación desde un nuevo currículo en la

Universidad de Guayaquil. Revista Universidad y Sociedad, 11(1), 243248.

Tapia-León, M., Rivera Villalta, M. D. C., Luján-Mora, S., \& Barros Bastidas, C. I. (2017). Análisis de la calidad de los resúmenes de tesis de grado de las universidades del Ecuador respecto a normas internacionales.

von Feigenblatt, Otto Federico, The Importance of Historical Heritage and the Fallacy of the Cancel Movement: International Case Studies (May 23, 2020). Journal of Alternative Perspectives in the Social Sciences (2020) Volume 10 No 3, 483-492, Available at SSRN: https://ssrn.com/abstract=

von Feigenblatt, Otto Federico, JAPÓN Y EL COVID-19 DESDE UN PUNTO DE VISTA SOCIOCULTURAL (Japan and COVID-19 from a Socio-cultural Perspective) (June 1, 2020). Revista Observatorio Iberoamericano de la Economía y la Sociedad del Japón (febrero 2020)., Available at SSRN: https://ssrn.com/abstract=3663848 von Feigenblatt, Otto Federico, The Significance of the Shang Period for the Contemporary Construction of Chinese Cultural Identity (April 10, 2020). Journal of Business and Entrepreneurial Studies, 4(2), 309-318. , Available at SSRN: https://ssrn.com/abstract= 